\title{
Occlusion of Vertebral Artery, Cerebellar Infarction and Obstructive Hydrocephalus following Cervical Spine Manipulation
}

\author{
Denis Cerimagic $^{a} \quad$ Josip Glavic ${ }^{a} \quad$ Arijana Lovrencic-Huzjan $^{b}$ Vida Demarin ${ }^{b}$ \\ ${ }^{a}$ Department of Neurology, General Hospital Dubrovnik, Dubrovnik, and ${ }^{\mathrm{b}}$ Department of Neurology, \\ 'Sestre Milosrdnice' University Hospital Zagreb, Zagreb, Croatia
}

Dear Sir,

The growing recognition of cervical manipulation as a treatment of neck pain and cervicogenic headaches has led to increased interest in potential complications that may result from this treatment approach. In 1947, Pratt-Thomas and Berger [1] reported 2 patients who died in less than $24 \mathrm{~h}$ after chiropractic treatment. Since their report, many authors have described patients with various neurological complications caused by cervical spine manipulations. Most of the patients described were relatively young, which probably reflects the age of people who seek this type of care. In this connection, complications mentioned most often are vertebral artery dissection and/or occlusion as well as consequential posterior circulation stroke [2]. To our knowledge, this is the first report of vertebral artery occlusion, cerebellar infarction and obstructive hydrocephalus as a result of cervical spine manipulation.

\section{Case Report}

The patient was a 46-year-old nonsmoking man with a previous history of neck pain. He was submitted to a complete checkup for life insurance purposes (including neurologist examination and carotid and vertebral artery color Doppler examination) 2 months before hospital admission, and the checkup results were normal. His personal and family anamnesis contained no information on risk factors of cerebrovascular disease nor any information on the existence of connective tissue disorder. Due to neck pain and cervicogenic headaches that lasted throughout several years, the patient was subjected to cervical spine manipulation.

The patient was admitted to hospital because of stitching interscapular pain accompanied by intense headache, nausea and vomiting which appeared $30 \mathrm{~h}$ following cervical manipulation. At admission to hospital, he was conscious and astatic. He had a severe horizontal nystagmus directed to the right and right-sided dysmetria. The Stewart-Holmes test (rebound phenomenon seen in cerebellar disorders) on the right arm was positive. His blood pressure was $140 / 80 \mathrm{~mm} \mathrm{Hg}$ and other vital signs were normal.

Complete hematology, biochemistry and coagulation tests were normal. The following analyses were performed: complement level, complement components $\mathrm{C} 3$ and $\mathrm{C} 4$, circulating immune complexes in blood, antiextractable nuclear antigen, antineutrophil cytoplasmatic antibodies, antistaphylolysin antibodies, antistreptolysin $\mathrm{O}$ test, lupus erythematosus cells, rheumatoid factor, antinuclear factor, and cerebrospinal fluid, and the findings were all normal. Serologic studies for syphilis, hepatitis and HIV were negative.
ECG, chest radiography, fundus oculi, transthoracic echocardiogram, brain CT scan and contrast-enhanced CT scan of the thoracic aorta were normal as well. Carotid and vertebral artery color Doppler examination revealed right vertebral artery occlusion (from the V2 segment distally).

The cervical collar ('Schanz') was applied and analgetic (tramadol), antiemetic (thiethylperazine) and prophylactic anticoagulant (low-molecular-weight heparin) therapies were introduced. During the following hours, the patient's vital functions were monitored. The values of blood pressure increased up to $230 / 130 \mathrm{~mm} \mathrm{Hg}$. Despite applied antiedematous (10\% solution of mannitol) and antihypertensive therapy (urapidil), the patient's intense headache and neck pain persisted. His state of consciousness worsened (Glasgow Coma Scale score $=6$ ). Because of acute respiratory insufficiency, he was placed on mechanical ventilation. The control brain CT scan after $18 \mathrm{~h}$ showed an extensive right cerebellar infarction (fig. 1a) including consequential obstructive hydrocephalus (fig. 1b). A neurosurgical implantation of external ventricular drainage was urgently performed. Following the operation, multislice CT angiography was performed, which confirmed the occlusion of the right vertebral artery (fig. 2). Skin biopsy was not performed. External ventric-

\section{KARGER}

Fax +4161306 1234 E-Mail karger@karger.ch www.karger.com
C 2007 S. Karger AG, Basel 0014-3022/07/0584-0248\$23.50/0

Accessible online at: www.karger.com/ene
Denis Cerimagic, MD

General Hospital Dubrovnik

Department of Neurology, Roka Misetica bb

HR-20000 Dubrovnik (Croatia)

Tel. +385 2043 1653, Fax +385 2042 6149, E-Mail deniscerimagic@yahoo.com 

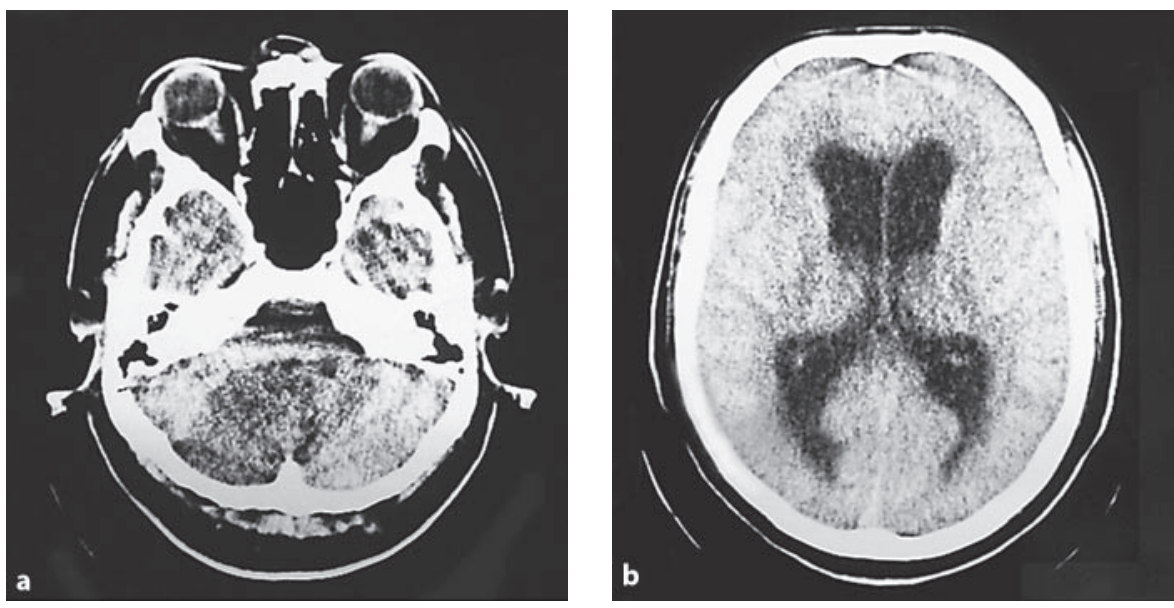

Fig. 1. Brain CT scan. a Right cerebellar infarction. b Hydrocephalus.

ular drainage was removed after 3 months. Four months afterwards, the patient's neurological examination showed mild rightsided dysmetria.

\section{Discussion}

Cervical spine manipulations take on the risk of many, potentially fatal neurological complications such as: vertebral artery dissection and/or occlusion [2], dissection and/or occlusion of the internal carotid artery $[2,3]$, occlusion of the basilar artery [4], posterior inferior cerebellar artery dissection [5], cerebellar and cerebral infarction [2], intracerebellar hematoma and obstructive hydrocephalus [6], brain stem infarction, for example, Wallenberg's syndrome [7], Déjérine syndrome [8], 'locked-in' syndrome [9]. The occurrence of radiculopathy and myelopathy [10] is also a possibility, as well as the occurrence of neuro-ophthalmological complications such as the Horner syndrome [2] and homonymous hemianopsia caused by occipital infarction [11]. Vascular complications are more frequent in the area of posterior (vertebrobasilar) than in the area of anterior circulation [10], and the vertebral artery dissection is especially important, most often localized at the level C1-C2 where the artery changes from its vertical course to a horizontal one [12].

It is difficult to estimate the incidence of spinal manipulative therapy complications, as they are probably underreported in the literature. Rothwell et al. [13] tested the association between recent cervical spine manipulation and vertebrobasilar accidents. They found that in the group of examinees younger than 45 years of age, vertebrobasilar dissection or occlusion was 5 times more likely than in controls. This corresponds to an incidence of 1.3 case of vertebrobasilar accidents among 100,000 individuals receiving cervical spine manipulation. A survey of 177 neurologists practicing in California revealed 55 strokes, 30 radiculopathies, and $16 \mathrm{my}$ elopathies resulting from spine manipulation during a 2-year period of time [10]. Spinal disk herniation or displacement of bony structures can result in spinal cord compression, radiculopathy, or cauda equina syndrome [10], but there is also a possibility of ischemic cervical myelopathy development in case of vertebral artery dissection [14].

In the case of multiple artery dissection, we must take into consideration a possible existence of connective tissue disorder such as the Marfan syndrome, the Ehlers-Danlos syndrome, fibromuscular dysplasia or cystic medial necrosis [3]. Predispositional factors for the development of complications from cervical spine manipulations are the following ones: advanced cervical spondylosis, atherosclerosis, stenosis of neck arteries, vasculitis (luetic arteritis), connective tissue disorder, congenital asymmetry of the posterior circulation and vertebrobasilar insufficiency $[3,5,10]$.

In our patient, we did not find any risk factor despite extensive diagnostic examination and our opinion is that vertebral ar-

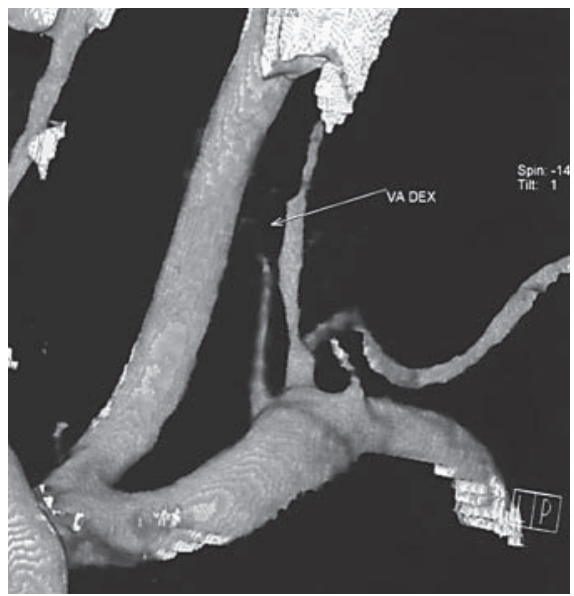

Fig. 2. Multislice CT angiography. Occlusion of the right vertebral artery (arrow).

tery occlusion is a result of dissection caused by hyperextension and neck rotation during cervical manipulation. Application of a cervical collar is not a routine procedure in such cases, and our intention was to prevent further propagation of the dissection.

Before cervical spine manipulation, it would be recommendable to perform color Doppler ultrasound examination of carotid and vertebral arteries, transcranial color Doppler imaging of the vertebrobasilar circulation and X-ray examination of the spine. It is impossible to completely exclude the risk of complication occurrence. The approach to each patient must be individual and in each separate case it is necessary to evaluate the benefit brought by cervical spine manipulation in relation to the potential risk that the patient is being exposed to.

\section{References}

1 Pratt-Thomas HR, Berger KE: Cerebellar and spinal injuries after chiropractic manipulation. JAMA 1947;133:600-603.

2 Jeret JS, Bluth M: Stroke following chiropractic manipulation. Report of 3 cases and review of the literature. Cerebrovasc Dis 2002;13:210-213.

3 Peters M, Bohl J, Thomke F, Kallen KJ, Mahlzahn K, Wandel F, Meyer zum Buschenfelde $\mathrm{KH}$ : Dissection of the internal carotid artery after chiropractic manipulation of the neck. Neurology 1995;45:2284-2287.

4 Carmody E, Buckley P, Hutchinson M: Basilar artery occlusion following chiropractic cervical manipulation. Ir Med J 1987;80:259-260. 
5 Sedat J, Dib M, Mahagne MH, Lonjon M, Paquis P: Stroke after chiropractic manipulation as a result of extracranial postero-inferior cerebellar artery dissection. J Manipulative Physiol Ther 2003;25:588-590.

6 Jacome DE: Isodense cerebellar hematoma. Neurology 1983;33:1201-1203.

7 Frumkin LR, Baloh RW: Wallenberg's syndrome following neck manipulation. Neurology 1990;40:611-615.

8 Yokota J, Amakusa Y, Tomita Y, Takabashi S: The medial medullary infarction (Dejerine syndrome) following chiropractic neck manipulation. No To Shinkei 2003;55:121-125.
9 Izquierdo-Casas J, Soler-Singla L, VivasDiaz E, Balaguer-Martinez E, Sola-Martinez I, Guimaraens-Martinez L: Locked in syndrome due to vertebral dissection and therapeutic options with intraarterial fibrinolysis in acute phase. Rev Neurol 2004;38:11391141.

10 Lee K, Philip MD, Walter G, McCormick GF Albers GW: Neurological complications following chiropractic manipulation: a survey of California neurologists. Neurology 1995; 45:1213-1215.

11 Gittinger JW Jr: Occipital infarction following chiropractic cervical manipulation. J Clin Neuroophthalmol 1986;6:11-13.
12 Saeed AB, Shuaib A, Al-Sulaiti G, Emery D: Vertebral artery dissection: warning symptoms, clinical features and prognosis in 26 patients. Can J Neurol Sci 2000;27:292-296.

13 Rothwell DM, Bondy SJ, Williams JI: Chiropractic manipulation and stroke: a population-based case-control study. Stroke 2001; 32:1054-1060.

14 Crum B, Mokri B, Fulgham J: Spinal manifestations of vertebral artery dissection. Neurology 2000;55:304-306. 